\title{
A 24 h Delay in the Redox Response Distinguishes the most Severe Stroke Patients from Less Severe Stroke Patients
}

\author{
Kimberly B Bjugstad ${ }^{1}$, Christopher Fanale ${ }^{2}$, Jeffrey Wagner ${ }^{2}$, Judd Jensen², Kristin Salottolo, Leonard T Rael ${ }^{1}$ and David Bar-Or ${ }^{1,3 *}$
}

${ }^{1}$ Trauma Research Department, Swedish Medical Center, Englewood, CO, USA

${ }^{2}$ NeuroSciences Department, Swedish Medical Center, Englewood, CO, USA

${ }^{3}$ Biomedical Sciences Department, Rocky Vista University, Aurora, CO and Penrose-St. Francis Health Services, Colorado Springs, CO, USA

\begin{abstract}
Objective: Admission measures of stroke severity are often used to assess 30-90 day outcomes. Since some patients have a poor outcome by discharge, we sought to identify a biomarker that distinguishes severities as well as acute outcomes. Disruptions in the equilibrium of the redox system were used as an indicator of stroke severity and acute outcome.
\end{abstract}

Methods: Oxidation reduction potential (ORP), a measure of the redox system, was assessed in plasma at admission and $24 \mathrm{~h}$ later in patients admitted with stroke symptoms $(n=76)$. Overall differences in ORP between stroke patients and healthy controls were determined. Within stroke patients, changes in ORP as a function of hospital discharge status, modified Rankin scale and NIHSS were assessed using ANOVA and the ability to predict acute outcome in ischemic stroke patients based on ORP was compared to NIHSS using Receiver Operator Characteristics.

Results: Stroke patients had higher ORP levels than healthy controls $(p<0.05)$. Patients discharged to hospice or who died had significantly lower values at admission than those discharged to home or intermediate care facilities $(p<0.05)$. However, in the following $24 \mathrm{~h}$, these patients significantly increased their ORP measures $(p<0.05)$. An ORP value $>146.6 \mathrm{mV}$ at admission was associated with a 46 fold increased odds of a good acute outcome.

Conclusion: ORP measured at admission identified patients which died or were discharged to hospice based on their lower ORP values. The lower ORP and subsequent increase $24 \mathrm{~h}$ later in the most severely affected patients may reflect a failure or a delay in engaging the redox system, a response that may, during acute stages, be beneficial.

Keywords: Ischemic stroke; Oxidative stress; Redox; NIHSS; Acute outcomes

\section{Introduction}

Approximately 795,000 people experience cerebral stroke per year in the USA [1]. Of those, $8-10 \%$ is expected to die in hospital $[1,2]$. Identifying patients most likely to have a fatal outcome during this acute period could assist health care providers in taking preventative actions.

Several stroke biomarkers have been used to predict 30-90 day outcomes based on serum levels of glial fibrillary acidic protein (GFAP), C-reactive protein (CRP), cortisol and glucose [3-5]. Only one study reported predictive values using in-hospital death as an end-point [6]. Serum glucose levels at admission reliably predicted $72 \mathrm{~h}$ mortality with a sensitivity of $88 \%$; however admission glucose levels could not predict 7 day mortality [6]. Since the average hospital length of stay for a stroke patient is 6 days [1], it would be beneficial to have a biomarker that includes the hospitalization period.

Data suggest stroke patients are under a state of oxidative stress $[7,8]$. Increases in reactive oxygen species (ROS), lipid peroxidation, isoprostanes and decreases in antioxidant activity were associated with functional measures like the Modified Rankin Scale (MRS) or the NIH Stroke Score (NIHSS) [7,8]. We used a novel technology to assess oxidative stress by measuring oxidation-reduction potential (ORP) in patients with stroke symptoms. ORP, also known as the redox potential, is an inclusive index measuring the activity of all oxidants to all antioxidants, providing an assessment of the entire redox system [9]. Previous studies using similar technology revealed changes in ORP as a result of exercise, traumatic brain injury and multi-trauma [10-12]. We hypothesized that there are reliable changes in the redox system after stroke reflected by differences in ORP as a function of stroke severity. Furthermore, these differences might be used for predicting acute outcome in stroke patients.

\section{Methods}

This study was conducted at Swedish Medical Center (SMC), a comprehensive stroke center located in Englewood, CO. Admitted patients who presented with stroke symptoms were consented into the study $(\mathrm{N}=162)$. Consent for participation was given by the patient or their legally authorized representative. The study was approved by the HealthOne Institutional Review Board and in accordance with institutional guidelines.

Patient inclusion criteria were consecutively admitted patients who presented with stroke symptoms between January 1, 2010 and December 31, 2011 and who were older or equal to 18 years of age. Patients were then excluded if they transferred into SMC $(n=63)$ from another facility, did not have a blood sample available on the day of admission $(\mathrm{n}=4)$ or did not have a stroke or did not have a transient ischemic attack (TIA) $(\mathrm{n}=19)$. Final study group included 76 patients.

*Corresponding author: David Bar-Or, Swedish Medical Center, Trauma Research Department, Room4-454, 501 East Hampden Avenue, Englewood, CO 80113 USA, Tel: 303-788-4089; Fax: 303-788-4064; E-mail: dbaror@ampiopharma.com

Received August 01, 2016; Accepted September 26, 2016; Published September 30, 2016

Citation: Bjugstad KB, Fanale C, Wagner J, Jensen J, Salottolo K, et al. (2016) A $24 \mathrm{~h}$ Delay in the Redox Response Distinguishes the most Severe Stroke Patients from Less Severe Stroke Patients. J Neurol Neurophysiol 7: 395. doi:10.4172/21559562.1000395

Copyright: (c) 2016 Bjugstad KB, et al. This is an open-access article distributed under the terms of the Creative Commons Attribution License, which permits unrestricted use, distribution, and reproduction in any medium, provided the original author and source are credited. 
Study procedures included up to once-daily collection of a blood sample during the patients' hospital stay, typically collected in the mornings. Venipuncture was carried out by a laboratory phlebotomist at the same time that blood was collected for diagnostic purposes. There were no other study procedures. Blood was processed to plasma, aliquoted and stored at $-80^{\circ} \mathrm{C}$.

\section{Oxidation reduction potential (ORP)}

ORP was measured using the RedoxSYS System (Aytu Bioscience, Englewood, CO), a galvanostatic-based technology [13]. The RedoxSYS analyzer, using a constant negligible current, measures the passive exchange of electrons between oxidants and reductants. This measure is the static ORP (sORP; millivolts, $\mathrm{mV}$ ); a higher sORP suggests an imbalance in the redox system in favor of oxidant activity, also known as oxidative stress. The analyzer then applies an increasing oxidative current which measures the induced exchange of electrons. This measures the capacity ORP (cORP; microcoulombs, $\mu \mathrm{C}$ ) which is the ability of the biological sample to withstand an oxidative insult, also known as antioxidant capacity. In the current study, cORP data $(\mu \mathrm{C})$ were not normally distributed, therefore, the inverse cORP (icORP, $\mu \mathrm{C}^{1}$ ) value was used, and thus a higher icORP suggests lower capacity to handle induced oxidative stress.

Both ORP measures were obtained from a single thawed roomtemperature plasma sample $(30 \mu \mathrm{L})$. Each sample was measured in duplicate. ORP values were also measured in archival plasma samples from 10 self-declared, non-smokers, healthy volunteers.

\section{Statistical analyses}

sORP and icORP values on day of admission and their $24 \mathrm{~h}$ change were used to determine if changes in the redox system could distinguish between various severities of stroke based on discharge status, discharge MRS scores and admission NIHSS. Hospital discharge status was categorized as follows: Home (home or home health), Intermediate (skilled nursing or rehabilitation sites) and Hospice/ Death. MRS scores were grouped based on actual values (0-6). NIHSS measures were assembled into four groups: scores 0-4: minor, 5-15: moderate, 16-20: moderately severe and 21-42: severe.

Additional variables of interest for ischemic stroke (IS) patients were etiology, tissue plasminogen activator (tPA) treatment and reperfusion therapy- intravenous and/or arterial thrombolysis (IV and/or IA). Age, sex, time between symptom onset and arrival and infectious complications were also analyzed in all stroke patients for differences in sORP and icORP at admission and $24 \mathrm{~h}$ change.

Data were analyzed using Student's t-test for two groups, ANOVA for more than two groups, Pearson's correlation, or Pearson's Chi square for proportions. Appropriate post-hoc analyses were done when a significant effect was found ( $\mathrm{p} \leq 0.05)$. A Receiver Operator Characteristic (ROC) statistic was used to identify admission sORP and NIHSS measures that best forecasted acute outcome dichotomized into a good acute outcome (patients discharged to home or intermediate care facilities) and poor acute outcome (patients transferred to hospice or who died in-hospital). The optimal cut-off criterion for predicting discharge status was identified using the Youden index for both variables. Data was analyzed using Statistica 13 (Dell, Inc.) and MedCalc Statistical Software 15.8. All statistics are presented within their respective graphs or within the text if not graphed. Data represent the mean \pm SEM or as otherwise noted in the tables.

\section{Results}

The final investigative group included 76 stroke patients; 49 with
IS, 9 with hemorrhagic stroke, and 18 with TIA. The average length of hospital stay for the home discharge group was 4 days, intermediate care group was 9.3 days and hospice/death group was 3.8 days. The prevalence of mortality (in-hospital death and those transferred to hospice) in the study group was $7.9 \%$, similar to previous reports [2]. General patient demographics are presented in Table 1.

Intra-patient reliability in ORP measures obtained from blood plasma samples was assessed by comparing replicate means and by correlation. There were no significant differences in $\mathrm{sORP}(\mathrm{t}(150)=1.10$, $\mathrm{p}>0.05)$ or in icORP $(\mathrm{t}(148)=0.51, \mathrm{p}>0.05)$ between replicates. The correlations between replicates were significant with $r=0.98\left(R^{2}=0.96\right.$, $\mathrm{p}<0.05)$ and $0.88\left(\mathrm{R}^{2}=0.77, \mathrm{p}<0.05\right)$ for sORP and icORP measures respectively. Because of the high level of reliability, further ORP analyses were performed on the mean value of the replicates.

\section{All stroke patients vs. control volunteers}

Archival control samples were significantly younger than the overall stroke population $(\mathrm{t}(84)=4.64, \mathrm{p}<0.001)$, thus stroke data were grouped into an age-matched stroke cohort and an unmatched older stroke cohort. Analyses of sORP and icORP values, using admission values for stroke cohorts, indicated that both age-matched and unmatched stroke patients had higher sORP and icORP values than controls $(p<0.05$; Figure 1 ) demonstrating that both stroke groups had elevated oxidative stress measures compared to normal controls.

There were no differences in SORP or icORP values between the

\begin{tabular}{|c|c|c|c|}
\hline & $\mathbf{N}$ & Mean \pm sem & $\begin{array}{c}\text { Median } \\
(\text { min-max })\end{array}$ \\
\hline Gender ( $\%$ male $)$ & $76(38.2 \%)$ & & \\
\hline Ethnicity (\% Caucasian) & $76(85.5 \%)$ & & \\
\hline Age (years) & 76 & $73.0 \pm 1.83$ & $78(26-94)$ \\
\hline Stroke Type & 76 & & \\
\hline $\begin{array}{l}\text { Hemorrhagic (hypertensive, } \\
\text { cerebral amyloid angiopathy, } \\
\text { aneurysm) }\end{array}$ & 9 & & \\
\hline Transient Ischemic Attack (TIA) & 18 & & \\
\hline Ischemic & 49 & & \\
\hline Cardioembolism & 28 & & \\
\hline $\begin{array}{l}\text { Large vessel atherosclerosis } \\
\text { (LVA) }\end{array}$ & 8 & & \\
\hline $\begin{array}{l}\text { Small vessel atherosclerosis } \\
\text { (SVA) }\end{array}$ & 5 & & \\
\hline $\begin{array}{l}\text { Other (vessel dissection, } \\
\text { cryptogenic, hypercoagulability) }\end{array}$ & 8 & & \\
\hline $\begin{array}{l}\text { Time from Symptom Onset } \\
\text { to Arrival }\end{array}$ & 68 & $4.36 \pm 0.92$ & $1.41(0.33-42.9)$ \\
\hline$<2 \mathrm{~h}$ & 48 & & \\
\hline $2-4 \mathrm{~h}$ & 19 & & \\
\hline $4-6 \mathrm{~h}$ & 5 & & \\
\hline $6-12 \mathrm{~h}$ & 4 & & \\
\hline$>12 \mathrm{~h}$ & 9 & & \\
\hline tPA treatment & 49 & & \\
\hline None & 32 & & \\
\hline Yes (time to treat in minutes) & 17 & $91.7 \pm 5.42$ & $88(61-135)$ \\
\hline Hospital length of stay (days) & 74 & $6.1 \pm 0.84$ & $4(1-44)$ \\
\hline NIHSS at admission & 64 & $7.3+0.88$ & $5(0-25)$ \\
\hline Infectious Complication & 95 & & \\
\hline No & 84 & & \\
\hline $\begin{array}{l}\text { Yes } \\
\text { (pneumonia, MRSE and/or UTI) }\end{array}$ & 11 & & \\
\hline
\end{tabular}

Table 1: Patient demographics and sample sizes (N). 
type of stroke or between the IS etiologies ( $\mathrm{p}>0.05$; Figures 2 and 3 and Table 1), thus at admission and after $24 \mathrm{~h}$, all stroke patients had similar levels of oxidative stress despite the type or cause of the stroke.

\section{Discharge status and mortality in all stroke patients}

Admission sORP values were higher in patients discharged to home or intermediate care facilities compared to those who died in-hospital or transferred to hospice ( $\mathrm{p}<0.05$; Figure $4 \mathrm{a}$ ), signifying a lower state of oxidative stress in this group with a poor acute outcome. In $24 \mathrm{~h}$, however, the death/hospice patients had a significant increase in sORP which was not observed in the other groups ( $<<0.05$; Figure $2 b)$. This change brought the poor acute outcome group's sORP values to the level measured in the other less severe group's $24 \mathrm{~h}$ later ( $p>0.05$, data not shown).

Admission icORP values were also higher in patients discharged to home or intermediate care $(\mathrm{p}<0.05$; Figure $4 \mathrm{c})$ compared to those who would be discharged to hospice or died. Higher icORP values are indicative of lower antioxidant capacity. In $24 \mathrm{~h}$, the hospice/death group again had a significant increase in their icORP values $(\mathrm{p}<0.05$; Figure $2 \mathrm{~d}$ ), such that by this time there were again no significant differences between the groups ( $p>0.05$, data not shown).

Because those with a poor acute outcome were significantly lower, ad-hoc analyses between the archived healthy controls and those who died or went to hospice were done and found no significant differences in admission sORP $(\mathrm{t}(14)=0.62, \mathrm{p}=0.54)$ but a significant increase $i \operatorname{CORP}(\mathrm{t}(14)=3.58, \mathrm{p}<0.001)$. This comparison would suggest that the fatal acute outcome patients were more similar to the control volunteers in sORP values than the other stroke patients at admission and their icORP tended to be in between the two groups.

Of the patients who died or went to hospice, the poor acute outcome group, $100 \%$ (6/6 patients; specificity) had an admission sORP $\leq 142.9 \mathrm{mV}$. Of those that went home or were discharged to an intermediate care facility, $78.6 \%$ (55/70 patients; sensitivity) had a sORP $>142.9 \mathrm{mV}$. The odds ratio that sORP values at admission $\leq 142.9$ $\mathrm{mV}$ would result in a good outcome was $46.5(\mathrm{p}<0.005)$. ROC analysis for this cut-off covered a significant area under the curve (AUC) of 0.92 for all stroke patients and 0.89 for the IS and TIA subgroup $(\mathrm{p}<0.05)$. Based on these data and similar reported prevalence rates $[1,2]$, we calculated the predictive values for estimating a non-fatal discharge status (home or intermediate care) for all patients and for IS/TIA patients with the result that both were $100 \%$ (Table 2). Since the length of stay in the hospital after admission for each group ranged from 1-44 days for home/intermediate care and 1-8 days for hospice/death, sORP measured at admission predicted a non-fatal acute outcome $24 \mathrm{~h}$ and up to 44 days, in advance.

\section{Stroke severity}

All stroke patients with a discharge MRS of 5-6 had lower sORP and icORP values at admission than those with scores $0-4(\mathrm{p}<0.05$; Figure 5). Thus, patients with the most severe strokes had low levels of oxidative stress (sORP) and better antioxidant capacities (icORP) initially. In $24 \mathrm{~h}$, patients with MRS of 5-6 had a large elevation in both sORP and icORP (Figure 5), which brought the values up to similar levels as those in the MRS groups 0-4 within the following $24 \mathrm{~h}$ ( $\mathrm{p}>0.05$, data not shown).

In the IS and TIA subgroup, the most severe stroke group (NIHSS 21-42) also had lower admission sORP and icORP values compared to the minor, moderate, or moderately severe groups $(\mathrm{p}<0.05$; Figure 6$)$. The moderately severe group had the highest sORP values at admission $(\mathrm{p}<0.05)$. During the next $24 \mathrm{~h}$, changes in sORP and icORP were not different; however enough change occurred in this time to permit the severe group to reach values that were comparable to the other groups.

To compare the predictive differences between sORP and NIHSS on acute outcome, a ROC analysis for NIHSS was also performed. The AUC for NIHSS taken at admission was $0.86(\mathrm{p}<0.05)$ and was not significantly different from the AUC found for $s O R P(p>0.05)$. A cutoff NIHSS value of $\leq 6$ was identified as the optimal criterion. A $100 \%$ of those with a poor acute outcome ( $3 / 3$; specificity) were above this cutoff but only $63.9 \%$ of those with a good acute outcome were below it (39/61; sensitivity). Overall, the positive and negative predictive values were similar but NIHSS was slightly less accurate than admission sORP values (Table 2).
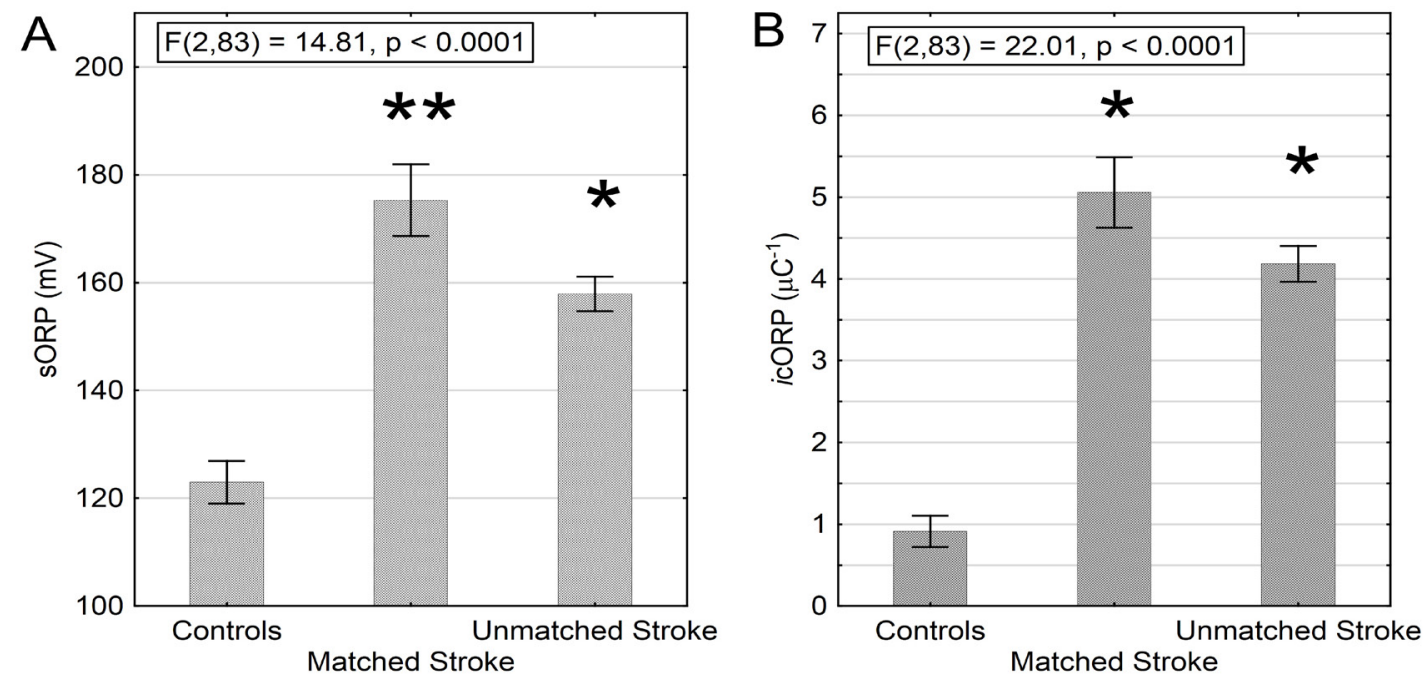

Figure 1: Stroke patients (all types) had higher sORP and icORP values than controls. Stroke patients age-matched to controls had higher sORP (A) and icORP values (B) Unmatched stroke patients, which were older, were also higher than controls values. The younger matched stroke patients had significantly higher sORP values than the older unmatched stroke patients, but they were not different from each other in icORP. Sample sizes were Controls $(n=10)$, Matched Stroke $(n=18)$ and Unmatched Stroke $(n=61){ }^{*}$ significantly greater than Controls; ${ }^{* *}$ significantly greater than Controls and Unmatched Stroke; $p<0.05$ 
Citation: Bjugstad KB, Fanale C, Wagner J, Jensen J, Salottolo K, et al. (2016) A 24 h Delay in the Redox Response Distinguishes the most Severe Stroke Patients from Less Severe Stroke Patients. J Neurol Neurophysiol 7: 395. doi:10.4172/2155-9562.1000395
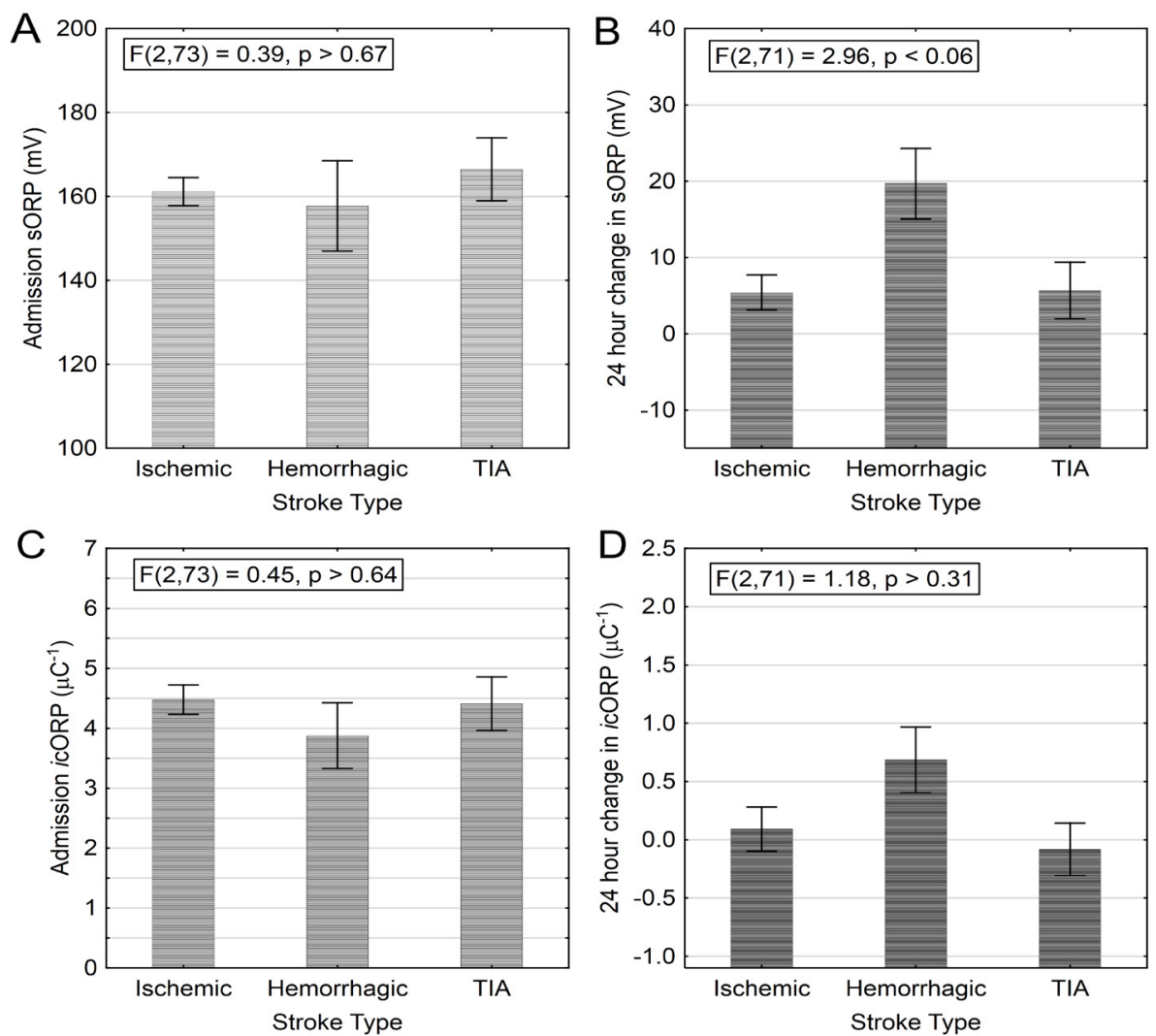

Figure 2: Different types of stroke had similar levels of oxidative stress. A: sORP values at admission were similar between ischemic, hemorrhagic, and TIA patients. $\mathrm{B}:$ In $24 \mathrm{~h}$, the changes in sORP were not significantly different although there was a trend for the hemorrhagic patients have elevated sORP values more so that the other groups. C/D: icORP did not differentiate between stroke types at admission or in the subsequent $24 \mathrm{~h}$.


Figure 3: Differences in the redox measures did not discriminate between different IS etiologies. A/B. IS patients with a cardioembolism (Card), large vessel atherosclerosis (LVA), small vessel atherosclerosis (SVA), or other source (i.e., vessel dissection, hypercoagulation etc.) had similar measures of sORP at admission and which did not change much in the succeeding $24 \mathrm{~h}$. C/D. icORP values also changed very little between groups at admission and in the next $24 \mathrm{~h}$. 

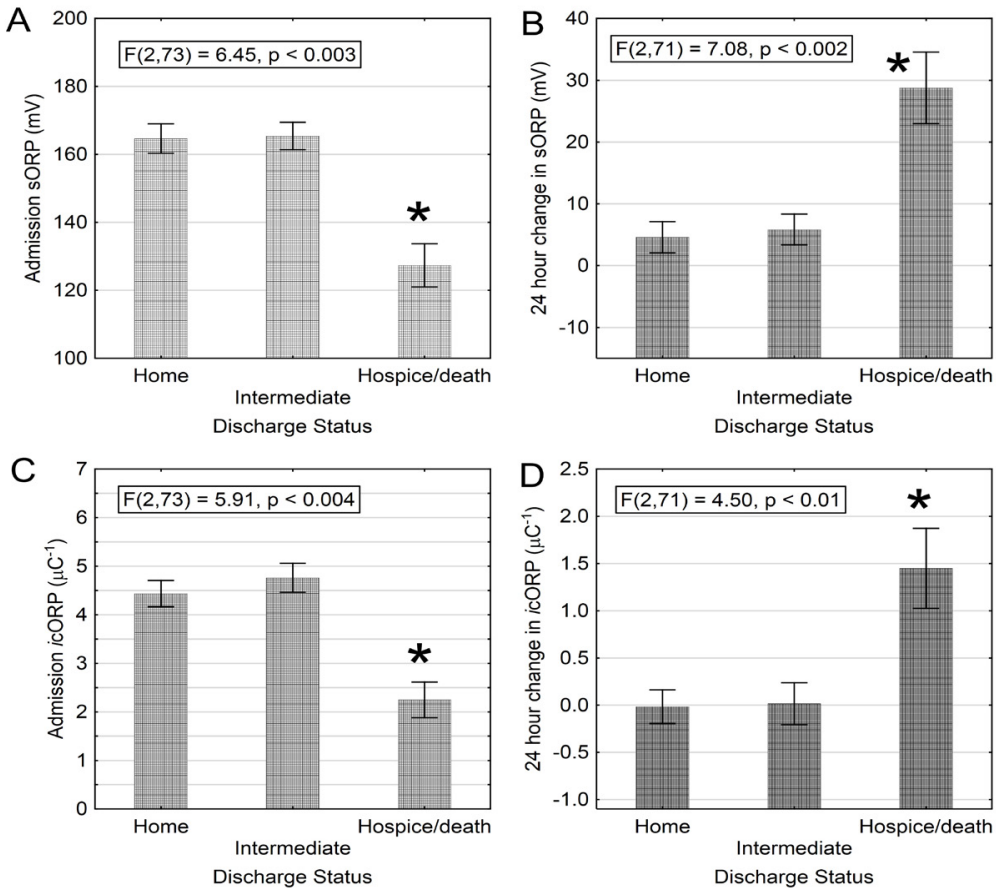

Figure 4: An acute outcome of hospice/death was related to diminish oxidative stress at admission for all stroke patients. A: Patients discharged to hospice or who died had lower sORP values at admission than patients with a better outcome, discharged to home or an intermediate care facility. B: A significant increase in sORP values occurred in the next $24 \mathrm{~h}$ in that same poor outcome group. C: icORP values at admission were also lower for the patients experiencing hospice/death at discharge compared to the other groups, indicating a higher level of antioxidant capacity at admission. D: Changes in icORP indicate that those discharged to hospice or who died in-hospital had an increase not measured in the other stroke patients, suggesting a loss of capacity in the next $24 \mathrm{~h}$. Relevant statistics are presented within each graph. Sample sizes were Home $(n=40)$, Intermediate $(n=30)$, Hospice/Death $(n=6){ }^{*}$ significantly different from all other groups, $p<0.05$

\begin{tabular}{|c|c|c|c|}
\hline & \multicolumn{2}{|c|}{ Admission sORP } & \multirow{2}{*}{$\begin{array}{c}\text { Admission NIHSS } \\
\text { IS and TIA Patients }(\mathrm{N}=64)\end{array}$} \\
\hline & All Stroke Patients ( $N=76)$ & IS and TIA Patients $(\mathrm{N}=67)$ & \\
\hline AUC $(95 \% \mathrm{Cl})$ & $\begin{array}{c}0.92 \text { * } \\
(0.83-0.97)\end{array}$ & $\begin{array}{c}0.89 \text { * } \\
(0.79-0.95)\end{array}$ & $\begin{array}{c}0.86 \text { * } \\
(0.75-0.93)\end{array}$ \\
\hline Sensitivity $(95 \% \mathrm{Cl})$ & $\begin{array}{c}78.6 \\
(67.1-87.5)\end{array}$ & $\begin{array}{c}78.1 \\
(66.0-87.5)\end{array}$ & $\begin{array}{c}63.9 \\
(50.6-75.8)\end{array}$ \\
\hline Specificity $(95 \% \mathrm{Cl})$ & $\begin{array}{c}100 \\
(54.1-100)\end{array}$ & $\begin{array}{c}100 \\
(29.2-100)\end{array}$ & $\begin{array}{c}100 \\
(29.2-100)\end{array}$ \\
\hline Positive Predictive Values $(95 \% \mathrm{Cl})$ & $\begin{array}{c}100 \\
(93.5-100)\end{array}$ & $\begin{array}{c}100 \\
(92.9-100)\end{array}$ & $\begin{array}{c}100 \\
(91.0-100)\end{array}$ \\
\hline Negative Predictive Value $(95 \% \mathrm{Cl})$ & $\begin{array}{c}28.6 \\
(11.3-52.2)\end{array}$ & $\begin{array}{c}17.6 \\
(3.8-43.4)\end{array}$ & $\begin{array}{c}12.0 \\
(2.5-31.2)\end{array}$ \\
\hline Accuracy & $80 \%$ & $79 \%$ & $66 \%$ \\
\hline
\end{tabular}

Table 2: Comparison of ROC analyses between sORP cutoff at admission (>142.9 mV) and the NIHSS cut off $(\leq 6)$ for identifying and predicting a positive outcome at time of hospital discharge (home or intermediate care).

\section{tPA Treatment, reperfusion and remaining variables}

TPA treatment was given to 17 IS and two TIA patients, with a mean time to treat of $92 \mathrm{~min}$ (min-max: 61-135 $\mathrm{min}$ ). sORP at admission and its $24 \mathrm{~h}$ difference did not change as a function of tPA treatment (Figure 7). Admission icORP values also did not distinguish between those given tPA and those that were not. However, in those that did get $\mathrm{PA}$ treatment, a significantly negative change in icORP during the subsequent $24 \mathrm{~h}$ was measured $(\mathrm{p}<0.05)$, suggesting an acquisition of antioxidant capacity in $24 \mathrm{~h}$ compared to a loss or no change in antioxidant capacity in the untreated stroke group. The distribution of tPA use was proportionate and met expected frequencies across IS etiologies, NIHSS groups, MRS and discharge statuses $\left(\mathrm{x}^{2}=1.96,7.56\right.$, $4.15,0.05$ respectively, $\mathrm{p}>0.05$ for all) suggesting no interactive effects with the above results.
Nineteen patients also received some type of reperfusion therapy, either intravenous thrombolysis (IV; $\mathrm{n}=14)$, intra-arterial thrombolysis (IA; $n=2$ ) or both (IA/IV; $n=3$ ). At admission, there no differences in sORP or icORP values between treatment groups (Figure 8 ). After treatment, during the subsequent $24 \mathrm{~h}$, there were significant changes with patients that received any kind of IA therapy with an increase in sORP value, whereas those with no therapy or IV only had minimal or no change in sORP. In contrast, those given IV therapy had a significant drop in icORP values in the subsequent $24 \mathrm{~h}$, signifying a gain in antioxidant capacity (Figure 8). The distribution of patients receiving reperfusion therapy was proportionate across discharge, MRS, ischemic etiology, and NIHSS groups $\left(x^{2}=2.13,9.03,6.03\right.$ and 12.26 , respectively, $p>0.05$ all), suggesting that the small changes induced by reperfusion therapy did not alter acute outcomes.

Differences in sORP and icORP were not significant as a function 

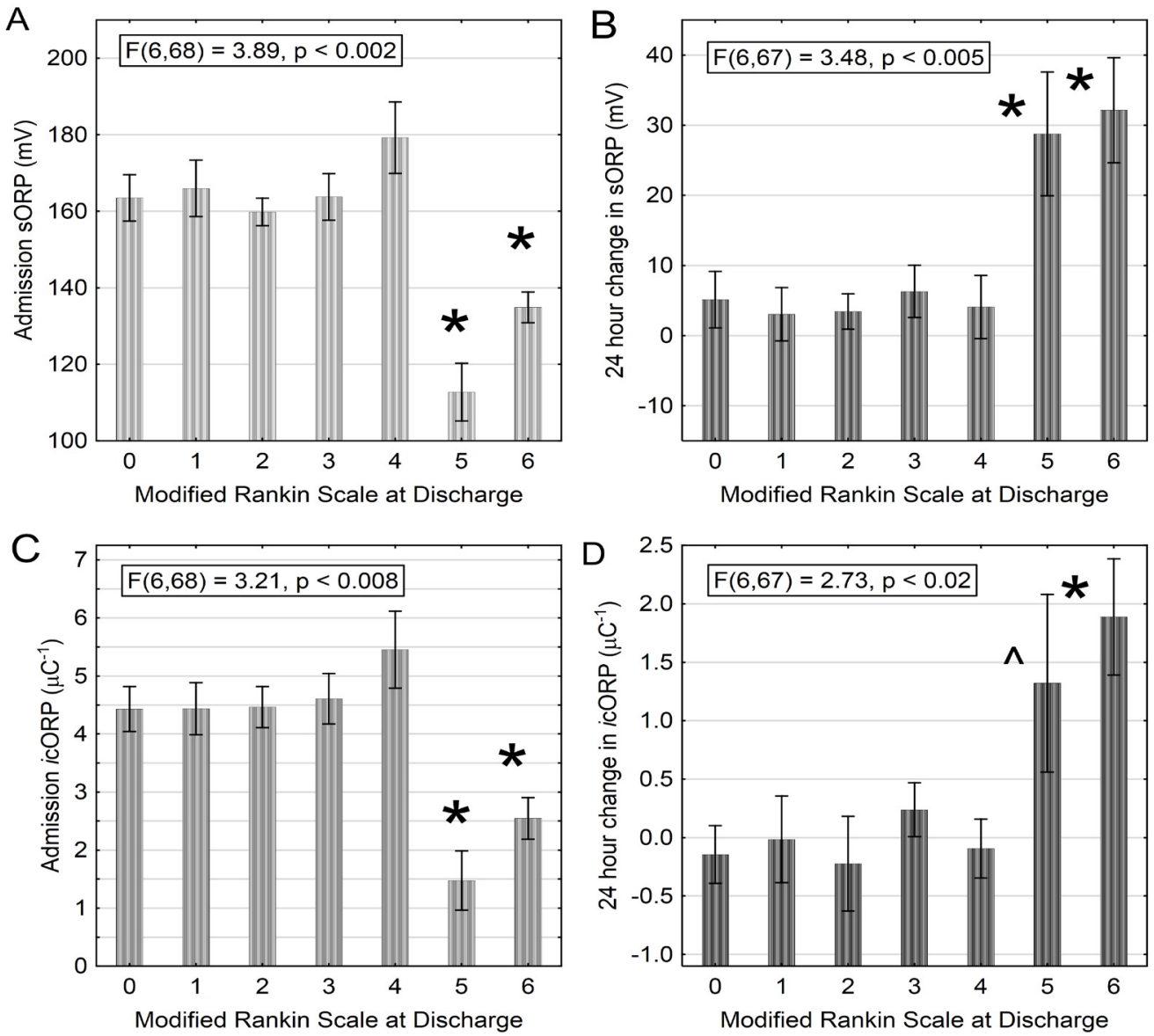

Figure 5: The highest MRS scores had the lowest measures of oxidative stress at admission. A: All stroke patients with a discharge MRS score of 5 or 6 had lower sORP values at admission than those with MRS scores between 0-4. B: In $24 \mathrm{~h}$, those with the highest MRS score (5-6) had a significant elevation in sORP measures. C: Antioxidant capacity was higher in those with an MRS score of 5 or 6 , indicated by lower icORP values compared to those with an MRS score of $0-4$. D: As with SORP, $24 \mathrm{~h}$ resulted in a significant loss in antioxidant capacity in the most severe patients, as revealed by increased in icORP values for those with MRS score of 5 or 6 . Sample size based on MRS scores $0(n=20), 1(n=13), 2(n=11), 3(n=15), 4(n=19), 5(n=3), 6(n=4)$. Relevant statistics are presented within each graph. ${ }^{\wedge}$, significantly different from Scores 0 and $2 ;{ }^{*}$ significantly different from all other scores; $p<0.05$.

of sex, time between symptom onset and arrival, or infectious complications ( $\mathrm{p}>0.05$ for all; data not shown). These variables were also proportionately distributed across discharge status $\left(\mathrm{x}^{2}=0.67,4.83\right.$ and 1.25 respectively, $\mathrm{p}>0.05$ for all).

\section{Discussion}

This study supports two major conclusions. First, the most severely affected stroke patients (death/hospice, MRS 5-6 and/or NIHSS $\geq 21$ ) have lower measures of oxidative stress at admission than less severe stroke patients, suggesting these patients are not under the state of oxidative stress that would be expected. However, the $24 \mathrm{~h}$ change in sORP and icORP suggests a delayed redox system response to stroke in the most severely affected stroke patients, as both ORP values increased during this time. Secondly, that difference in admission sORP values could project future discharge status (acute outcomes) of stroke patients.

Contrary to traditional views, a redox imbalance in favor of oxidative stress can be beneficial [14-16] and failure or a delay in engaging the redox system to oxidative stress may be an important indicator of outcome [17]. In terms of stroke, oxidative stress has important roles in vascular remodeling and inflammatory responses.
Cerebral vascular tone is adjusted through the production of oxygenbased radicals via the activity of NADPH-oxidase enzymes (NOX) [18-20]. In rat models of stroke, NOX, and thus oxygen-based radicals, increase within hours of occlusion and co-localize with anigiogenesis $[20,21]$. A delayed response in the redox system, as measured in our most severely affected stroke patients, could result in postponed vascular and redox compensations thus increasing ischemic brain damage and consequentially in poor acute outcomes [22].

The generation of oxidative stress and its modulation of the inflammatory response is commonly viewed as a deleterious cycle. Most studies report that elevations in the immune response are associated with higher NIHSS values and poorer outcomes however these studies omitted the most severe stroke patients by looking at longterm outcomes and thus removing those patients who died in hospital or by grouping NIHSS moderately severe and severe groups together (NIHSS $\geq 14)[4,23,24]$. It is possible that they missed observing changes in the immune system on the most severe stroke patients with the poor acute outcomes. Several studies without these limitations have shown that those patients with the highest NIHSS at admission and largest infarct volumes initially had significantly lower monocyte HLA-DR expression, fewer T-cells, fewer T-helper cells, fewer cytotoxic T-cells and lower interferon gamma (INF- $\gamma$ ) expression and elevated cortisol 

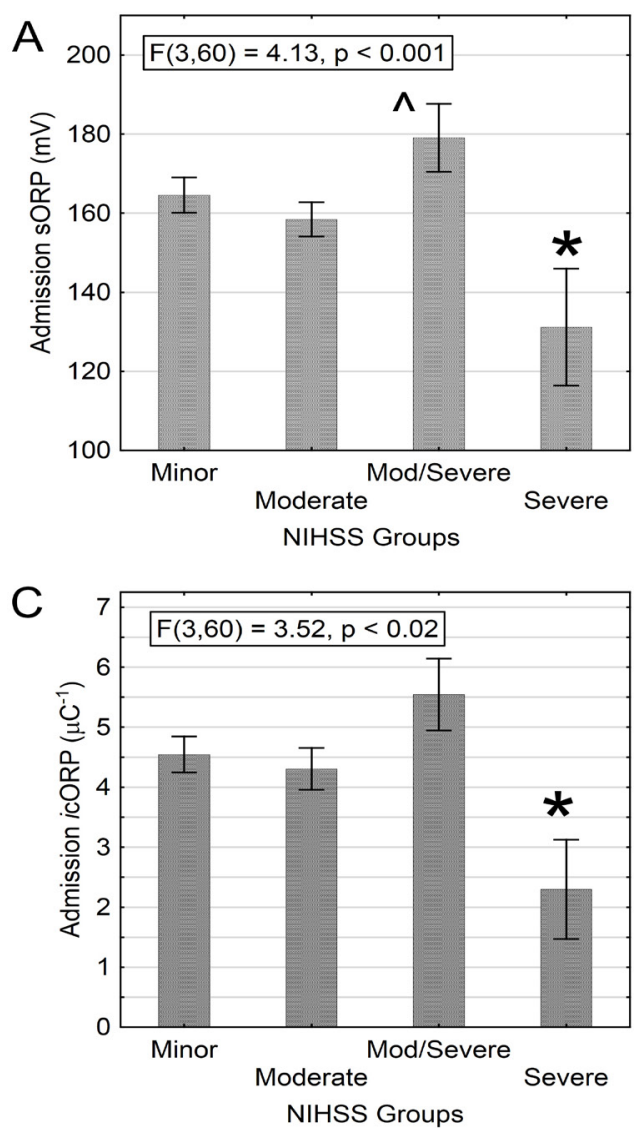
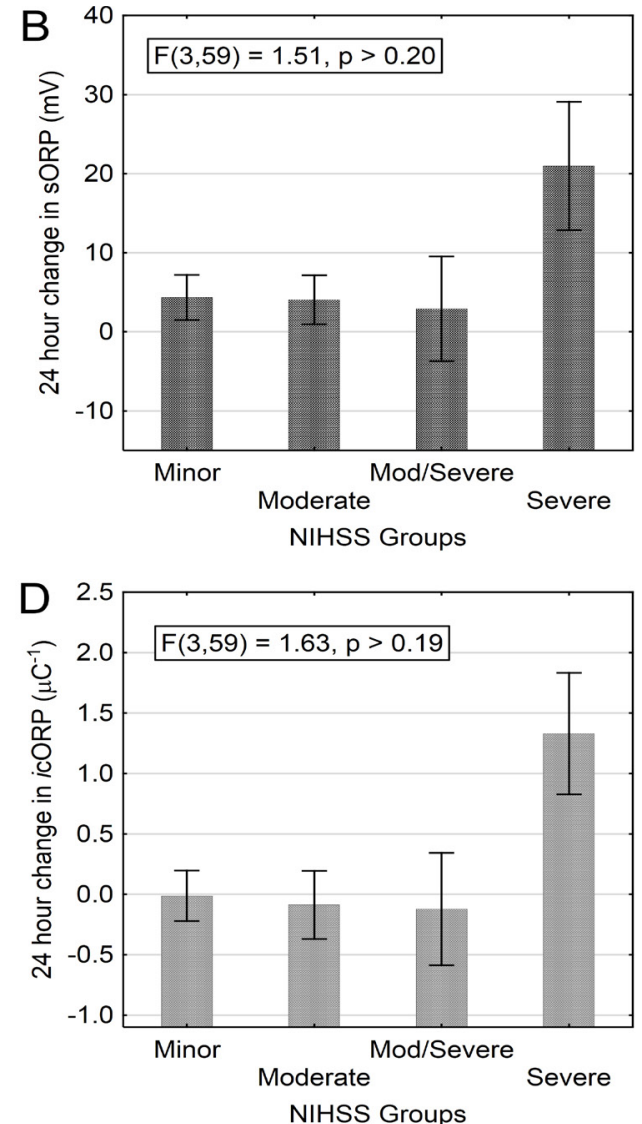

Figure 6: In IS patients, these were NIHSS group $(\geq 21)$ had lower measures of oxidative stress than the less severe NIHSS groups. A: IS and TIA patients with NIHSS scores falling into the severe level (NIHSS $\geq 21$ ) had significantly lower sORP values, whereas those with a moderately severe (mod/severe; NIHSS 16-20)) level had slightly higher sORP values. B: While there was some change in sORP in $24 \mathrm{~h}$ post-admission, this did not reach significance. C: The severe stroke group also had smaller admission icORP values than the other groups. D: Changes in icORP during the $24 \mathrm{~h}$ period post-admission did not reach significance. Sample sizes based on NIHSS groups were Minor (NIHSS 0-4; $=28$ ), Moderate (NIHSS 5-15; $n=24$ ), Mod/Severe (NIHSS 16-20; $n=8$ ) and Severe (NIHSS $\geq 21 ; n=4$ ). Relevant statistics are presented within each graph. ^significantly greater than Severe and Moderate groups; * ${ }^{*}$ ignificantly different from all other groups, $p<0.05$.

$[5,25,26]$. In the current data set, an attenuation of the oxidative stress response was recorded and because ROS modulates the immune response, it is possible that a low SORP at admission could be an early indicator for immunodepression in stroke [27,28].

As an indicator of the redox system, the initial sORP levels provided insight into the outcome of stroke patients, with those having higher sORP values at admission, greater than $142.4 \mathrm{mV}$, most likely to have a more favorable acute outcome (discharge to home or intermediated facility) compared to those with a low sORP (less than 142.4) having a poor acute outcome (discharge to hospice or death). In our study, we compared the predictive ability of sORP admission levels to admission NIHSS and found that SORP had better predictive statistics, including a better accuracy (Table 2). Earlier work evaluating the predictive power of NIHSS on outcome has produced similar values found to the current study, with AUCs between 0.78 and $0.90,88-91 \%$ of good outcomes correctly identified and $69-88 \%$ of poor outcomes identified [29-32]. The variability in the NIHSS predictive power between studies most likely reflects different NIHSS cut-off values and differentially defined outcomes, although it is possible that the variability lies in the administration of the NIHSS. Administration of the NIHSS requires a certified rater, yet despite this, there can be large variation in scoring with ataxia and facial weakness having the highest rate of subjective interpretation [33,34]. Measuring sORP is objective and requires no special certification, thus the fact that the sORP is at least as reliable as NIHSS and at most more reliable than NIHSS, measuring sORP is an unbiased and accessible indicator of acute outcome in stroke.

Biomarkers, GFAP, CRP, cortisol and glucose, are all unbiased measures that have been used to predict post-hospital (30-90 days) mortality [3-5] rather than more acute, in-hospital outcomes. Since $8-10 \%$ of stroke patients will die in-hospital $[1,2]$ and $84 \%$ of hospice patients die with a median length of stay of 13 days [35] we felt that predicting early mortality was an important event. It is unknown if measuring GFAP or CRP could predict a more immediate outcome, however, these markers are not adapted to the rapid turnaround times needed to estimate in-hospital mortality. The potential prognostic value provided by sORP measures could be used in the decision making processes regarding treatment with the intent to prevent predicted mortality.

Unfortunately, how to proceed and with what treatment is still debated. TPA, an accepted FDA approved drug for IS, has a $3 \mathrm{~h}$ window of effectiveness from the time of symptom onset [36,37]. In the current study, $30 \%$ of IS patients were outside this window, only $36 \%$ received tPA and tPA treatment was not associated with a better acute outcome. There was an increase in antioxidant capacity after $\mathrm{PPA}$ treatment in the $24 \mathrm{~h}$ period after admission, however this change was not associated with acute outcomes. Previously, in less severe stroke patients, lower 
Citation: Bjugstad KB, Fanale C, Wagner J, Jensen J, Salottolo K, et al. (2016) A 24 h Delay in the Redox Response Distinguishes the most Severe Stroke Patients from Less Severe Stroke Patients. J Neurol Neurophysiol 7: 395. doi:10.4172/2155-9562.1000395
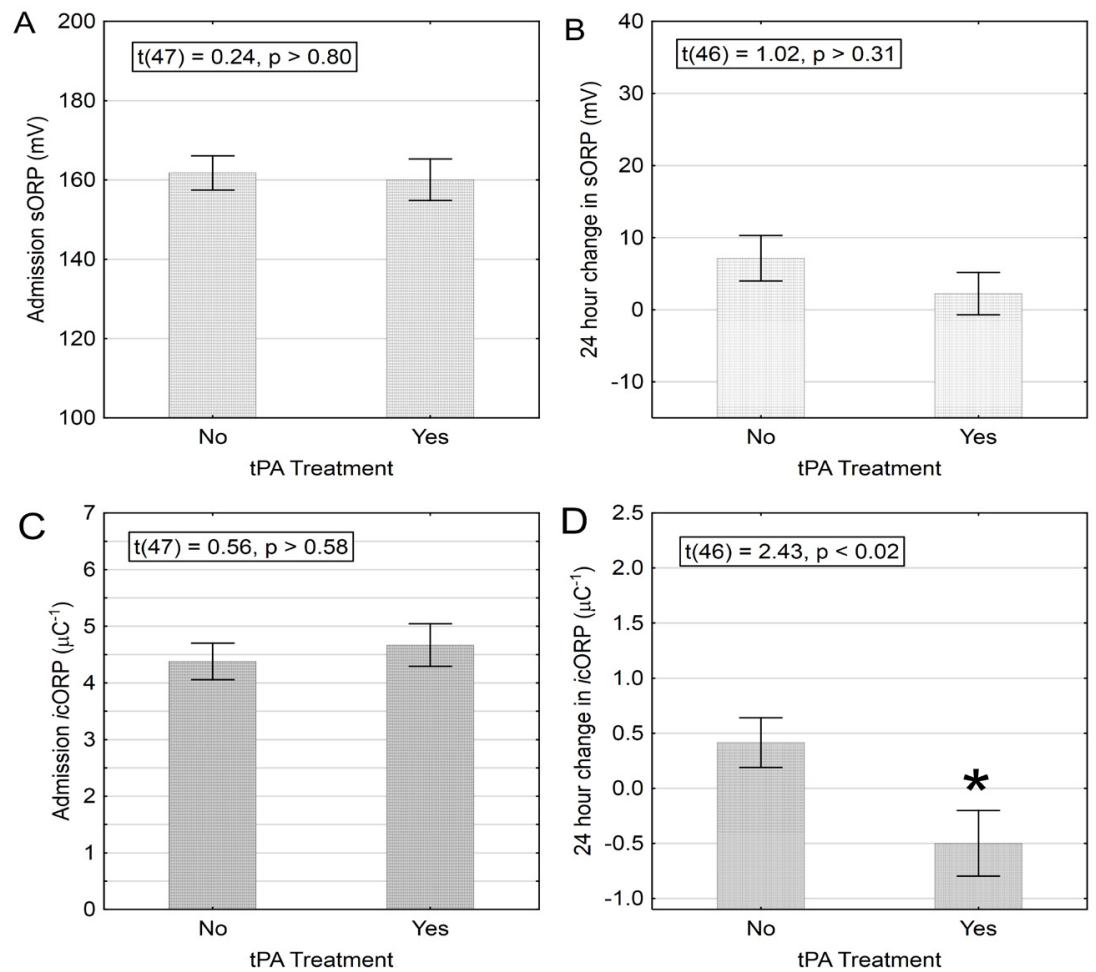

Figure 7: Antioxidant capacity increases in those given tPA treament the $24 \mathrm{~h}$ period after admission. A/B/C. sORP and icORP values were similar between those IS and TIA patients that received tPA treatment and there was no difference in how sORP changed in the following 24 hours. D. In those given tPA treatment, there was a significant decrease in icORP values, indicating an increase in antioxidant capacity during next $24 \mathrm{~h}$, which was not seen in those that were not given tPA treatment. *significantly less than the No tPA treatment group, $p<0.05$.
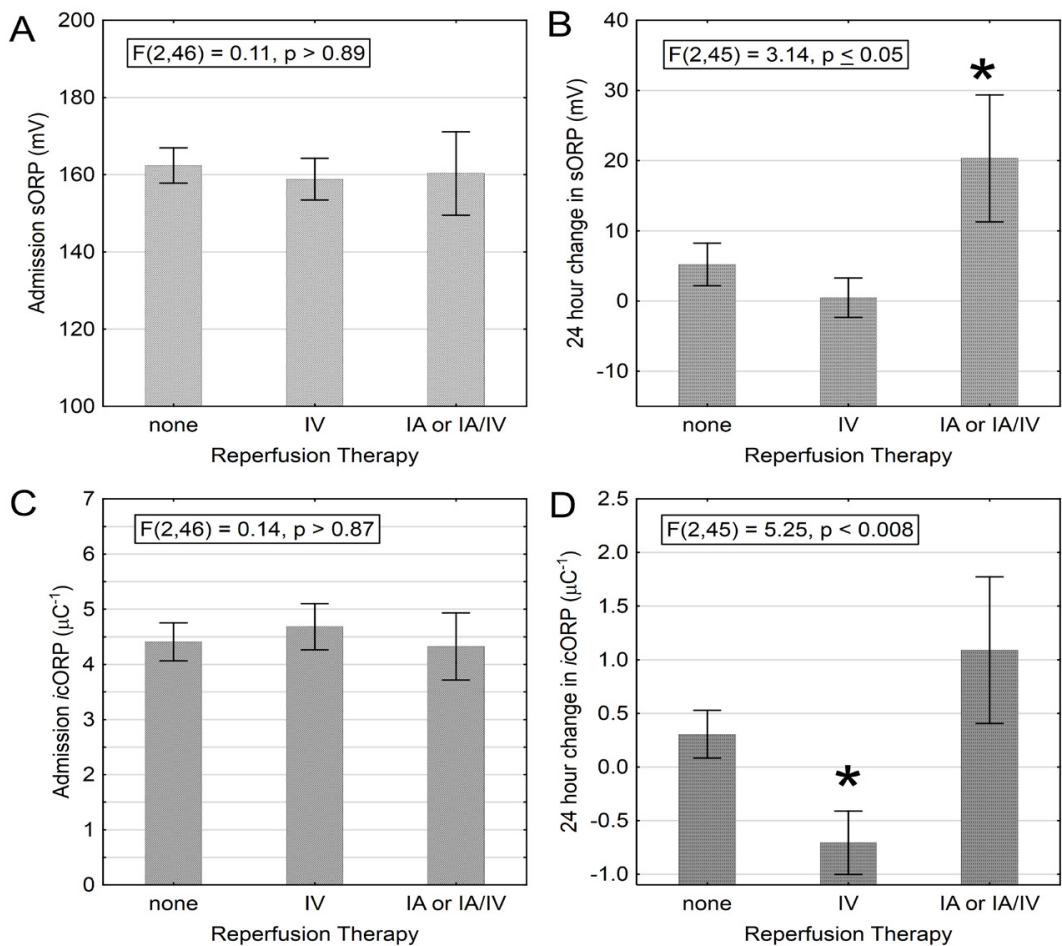

Figure 8: Reperfusion therapy changes the redox balance. A/C. There were no significant differences in admission ORP values between IS and TIA patients given IV, IA or IA and IV (IA/IV) reperfusion therapy from those that did not. B. $24 \mathrm{~h}$ after admission, those given IA or IA/IV therapy had a significant increase in sORP compared to IV or untreated patients. D. In contrast, those given IV therapy had a significant decrease in icORP values during the following $24 \mathrm{~h}$, suggesting a gain in antioxidant activity during this time. *significantly different from the remaining groups, $p<0.05$. 
oxidative stress and tPA treatment were associated with better longterm outcomes $[7,8,37]$. Similar to tPA treatment, IV reperfusion therapy in the current study was associated with increased antioxidant capacity in the period post-treatment, but it was not associated with a better acute outcome despite previous studies indicating its clear benefit in long-term outcomes [38].

Several studies report beneficial effects of antioxidant-based treatments given outside the tPA window, such as edaravone within 72 $\mathrm{h}$, ebselen within $24 \mathrm{~h}$ or lipophilic statins anytime between 1-10 days [39-42]. These treatments support the notion that reducing oxidative stress in stroke could be beneficial $[39,40,43]$. Our study confirms that in general, stroke patients are under a state of oxidative stress compared to healthy controls. Further, we endorse the idea that oxidative stress increases somewhat with stroke severity $[7,8]$; however, the most severe stroke patients, those discharged to hospice/death, with MRS of 5-6 or NIHSS scores $>21$ had lower sORP measures, compared with other less severe stroke patients. The fact that this has not been reported before might be due to previous studies investigating longer outcomes (30 or 90 days) [7,8,39-42], thereby eliminating the most severely affected stroke patients- those who die in-hospital or shortly thereafter. Therefore, studies suggesting that antioxidant treatment may benefit stroke patients are limited to those with a non-fatal acute outcomes and it remains unknown if it would benefit or harm patients with the most severe stroke and delayed redox response.

\section{Conclusion}

Data suggests that the redox system may need to favor oxidative stress at the outset of stroke to have a better acute outcome at hospital discharge. The failure to generate a timely oxidative stress response could result in a poor or fatal outcome by hospital discharge, as our data suggests that low sORP at admission is associated with poor acute outcomes. Once outside the acute outcome window, high oxidative stress does seem to be associated with poor post-hospital outcomes ( $\geq 30$ days). Finally, treatment decisions, especially if considering an antioxidant approach, ought to be made with the understanding of the oxidative state of the patient prior to administration, as these therapies are currently untested in the most severe stroke patients with a low or delayed oxidative stress response.

\section{Acknowledgement}

The authors gratefully acknowledge the collection of patient samples and maintenance of patient records by Rachel Aumann, R.N. and Anita Leyden, R.N. This study was supported by Trauma Research, L.L.C. (Englewood, CO), the Swedish Medical Center (Englewood, CO), St. Anthony Central Hospital (Denver $\mathrm{CO}$ ), and the Institute for Molecular Medicine (Englewood, CO). Dr. Bjugstad is a paid employee and Dr. Bar-Or is a consultant for Aytu BioScience, Inc. The remaining co-authors declare no competing interests.

\section{References}

1. Mozaffarian D, Benjamin EJ, Go AS, Arnett DK, Blaha MJ, et al. (2015) Heart disease and stroke statistics--2015 update: A report from the American Heart Association. Circulation 131: e29-322.

2. Ovbiagele B (2010) Nationwide trends in in-hospital mortality among patients with stroke. Stroke 41: 1748-1754.

3. Xiong L, Yang Y, Zhang M, Xu W (2015) The use of serum glial fibrillary acidic protein test as a promising tool for intracerebral hemorrhage diagnosis in Chinese patients and prediction of the short-term functional outcomes. Neurol Sci 36: 2081-2087.

4. Rocco A, Ringleb PA, Grittner U (2015) Follow-up C-reactive protein level is more strongly associated with outcome in stroke patients than admission levels. Neurol Sci 36: 2235-2241.

5. Zi WJ and Shuai J (2013) Cortisol as a prognostic marker of short-term outcome in Chinese patients with acute ischemic stroke. PLoS One 8: e72758.
6. Nardi K, Milia P, Eusebi P, Paciaroni M, Caso V, et al. (2012) Predictive value of admission blood glucose level on short-term mortality in acute cerebral ischemia. J Diabetes Complications 26: 70-76.

7. Nanetti L, Raffaelli F, Vignini A, Perozzi C, Silvestrini M, et al. (2011) Oxidative stress in ischaemic stroke. Eur J Clin Invest 41: 1318-1322.

8. Seet RC, Lee CY, Chan BP, Sharma VK, Teoh HL, et al. (2011) Oxidative damage in ischemic stroke revealed using multiple biomarkers. Stroke 42: 2326-2329.

9. Costantini D, Verhulst S (2009) Does high antioxidant capacity indicate low oxidative stress? Functional Ecology 23: 506-509.

10. Rael LT, Bar-Or R, Salottolo K, Mains CW, Slone DS, et al. (2009) Injury severity and serum amyloid A correlate with plasma oxidation-reduction potential in multi-trauma patients: A retrospective analysis. Scand J Trauma Resusc Emerg Med 17: 57

11. Rael LT, Bar-Or R, Mains CW, Slone DS, Levy AS, et al. (2009) Plasma oxidation-reduction potential and protein oxidation in traumatic brain injury. $\mathrm{J}$ Neurotrauma 26: 1203-1211.

12. Stagos D, Goutzourelas N, Bar-Or D, Ntontou AM, Bella E, et al. (2015) Application of a new oxidation-reduction potential assessment method in strenuous exercise-induced oxidative stress. Redox Rep 20: 154-162.

13. Rael LT, Bar-Or R, Kelly MT, Carrick MM, Bar-Or D (2015) Assessment of oxidative stress in patients with an isolated traumatic brain injury using disposable electrochemical test strips. Electroanalysis 27: 2567-2573.

14. Scudellari M (2015) The science myths that will not die. Nature 528: 322-325.

15. Watson JD (2014) Type 2 diabetes as a redox disease. Lancet 383: 841-843.

16. Teodoro JS, Rolo AP, Palmeira CM (2013) The NAD ratio redox paradox: Why does too much reductive power cause oxidative stress? Toxicol Mech Methods 23: 297-302.

17. Zhi L, Hu X, and Han C (2014) Biphasic changes (over reduction and over oxidation) of plasma redox status and clinical implications in early stage of severe burns. J Crit Care 29: 1063-1068.

18. Karimi Galougahi K, Ashley EA, Ali ZA (2016) Redox regulation of vascular remodeling. Cell Mol Life Sci 73: 349-363.

19. Miller AA, Drummond GR, Schmidt HH, Sobey CG (2005) NADPH oxidase activity and function are profoundly greater in cerebral versus systemic arteries. Circ Res 97: 1055-1062.

20. Taylor CJ, Weston RM, Dusting GJ, and Roulston CL (2013) NADPH oxidase and angiogenesis following endothelin-1 induced stroke in rats: Role for nox2 in brain repair. Brain Sci 3: 294-317.

21. Sobey CG, Heistad DD, Faraci FM (1997) Mechanisms of bradykinin-induced cerebral vasodilatation in rats. Evidence that reactive oxygen species activate $\mathrm{K}+$ channels. Stroke 28: 2290-2294.

22. Terpolilli NA, Kim SW, Thal SC, Kataoka H, Zeisig V, et al. (2012) Inhalation of nitric oxide prevents ischemic brain damage in experimental stroke by selective dilatation of collateral arterioles. Circ Res 110: 727-738.

23. Emsley HC, Smith CJ, Gavin CM, Georgiou RF, Vail A, et al. (2003) An early and sustained peripheral inflammatory response in acute ischaemic stroke: Relationships with infection and atherosclerosis. J Neuroimmunol 139: 93-101.

24. Idicula TT, Brogger J, Naess H, Waje-Andreassen U, Thomassen L (2009) Admission C-reactive protein after acute ischemic stroke is associated with stroke severity and mortality: The 'Bergen stroke study'. BMC Neurol 9: 18.

25. Urra X, Cervera A, Villamor N, Planas AM, Chamorro A (2009) Harms and benefits of lymphocyte subpopulations in patients with acute stroke. Neuroscience 158: 1174-1183.

26. Haeusler KG, Schmidt WU, Foehring F, Meisel C, Guenther C, et al. (2012) Immune responses after acute ischemic stroke or myocardial infarction. Int J Cardiol 155: 372-377.

27. Wink DA, Hines HB, Cheng RY, Switzer CH, Flores-Santana W, et al. (2011) Nitric oxide and redox mechanisms in the immune response. J Leukoc Biol 89: 873-891.

28. Nayernia Z, Jaquet $\mathrm{V}$, Krause KH (2014) New insights on NOX enzymes in the central nervous system. Antioxid Redox Signal 20: 2815-2837.

29. Jeng JS, Huang SJ, Tang SC, Yip PK (2008) Predictors of survival and 
Citation: Bjugstad KB, Fanale C, Wagner J, Jensen J, Salottolo K, et al. (2016) A 24 h Delay in the Redox Response Distinguishes the most Severe Stroke Patients from Less Severe Stroke Patients. J Neurol Neurophysiol 7: 395. doi:10.4172/2155-9562.1000395

functional outcome in acute stroke patients admitted to the stroke intensive care unit. J Neurol Sci 270: 60-66.

30. Mansour OY, Megahed MM, and Abd Elghany EHS (2015) Acute ischemic stroke prognostication, comparison between Glasgow Coma Score, NIHS Scale and Full Outline of UnResponsiveness Score in intensive care unit. Alexandria Journal of Medicine 51: 247-253.

31. Sablot D, Belahsen F, Vuillier F, Cassarini JF, Decavel P, et al. (2011) Predicting acute ischaemic stroke outcome using clinical and temporal thresholds. ISRN Neurol 2011: 354642

32. Schaefer PW, Pulli B, Copen WA, Hirsch JA, Leslie-Mazwi T, et al. (2015) Combining MRI with NIHSS thresholds to predict outcome in acute ischemic stroke: Value for patient selection. AJNR Am J Neuroradiol 36: 259-264.

33. Hills NK, Josephson SA, Lyden PD, Johnston SC (2009) Is the NIHSS certification process too lenient? Cerebrovasc Dis 27: 426-432.

34. Lyden P, Raman R, Liu L, Grotta J, Broderick J, et al. (2005) NIHSS training and certification using a new digital video disk is reliable. Stroke 36: 2446-2449.

35. Caffrey C, Sengupta M, Moss A, Harris-Kojetin L, Valverde R (2011) Home health care and discharged hospice care patients: United States, 2000 and 2007. Natl Health Stat Report: 1-27.

36. Hebert M, Lesept F, Vivien D, and Macrez R (2016) The story of an exceptional serine protease, tissue-type plasminogen activator (tPA). Rev Neurol (Paris) 172: $186-97$
37. Zivin JA (2009) Acute stroke therapy with tissue plasminogen activator (tPA) since it was approved by the U.S. Food and Drug Administration (FDA). Ann Neurol 66: 6-10

38. Hlavica M, Diepers M, Garcia-Esperon C, Ineichen BV, Nedeltchev K, et al. (2015) Pharmacological recanalization therapy in acute ischemic stroke evolution, current state and perspectives of intravenous and intra-arterial thrombolysis. J Neuroradiol 42: 30-46.

39. Otomo E (2003) Effect of a novel free radical scavenger, edaravone (MCl-186) on acute brain infarction. Randomized, placebo-controlled, double-blind study at multicenters. Cerebrovasc Dis 15: 222-229.

40. Flint AC, Kamel H, Navi BB, Rao VA, Faigeles BS, et al. (2012) Statin use during ischemic stroke hospitalization is strongly associated with improved post-stroke survival. Stroke 43: 147-154.

41. Song B, Wang Y, Zhao X, Liu L, Wang C, et al. (2014) Association between statin use and short-term outcome based on severity of ischemic stroke: A cohort study. PLoS One 9: e84389.

42. Yamaguchi T, Sano K, Takakura K, Saito I, Shinohara Y, et al. (1998) Ebselen in acute ischemic stroke: A placebo-controlled, double-blind clinical trial. Ebselen Study Group. Stroke 29: 12-17.

43. Amaro S, Llull L, Renú A, Laredo C, Perez B, et al. (2015) Uric acid improves glucose-driven oxidative stress in human ischemic stroke. Ann Neurol 77 775-783. 